ISSN 1996-1944

www.mdpi.com/journal/materials

Article

\title{
Corrosion Prevention of Aluminum Nanoparticles by a Polyurethane Coating
}

\section{Toshiyasu Nishimura* and Vedarajan Raman}

National Institute for Materials Science (NIMS), 1-2-1 Sengen, Tsukuba, Ibaraki 305-0047, Japan; E-Mail: raman@jaist.ac.jp

* Author to whom correspondence should be addressed; E-Mail: Nishimura.toshiyasu@ nims.go.jp.

Received: 28 January 2014; in revised form: 28 May 2014 / Accepted: 9 June 2014 /

Published: 19 June 2014

\begin{abstract}
In order to prevent corrosion, aluminum nanoparticles were coated with a polyurethane polymer. The coverage of the polyurethane polymer was controlled from 0 to $100 \%$, which changed the corrosion rate of the nanoparticles quantitatively. The surface of the polymer coating was investigated by Transmission Electron Microscopy (TEM) and Atomic Force Microscopy (AFM), and the corrosion resistance of the nanoparticles was estimated by a wet/dry corrosion test on a Pt plate with a $\mathrm{NaCl}$ solution. From a TEM with EDAX analysis, the 10 mass\% polymer coated Al particles in the synthesis were almost $100 \%$ covered on the surface by a polymer film of $10 \mathrm{~nm}$ thick. On the other hand, the 3 mass $\%$ polymer coated $\mathrm{Al}$ was almost $40 \%$ covered by a film. In the AFM, the potential around the $\mathrm{Al}$ particles had a relatively low value with the polymer coating, which indicated that the conductivity of the $\mathrm{Al}$ was isolated from the Pt plate by the polymer. Both the corrosion and $\mathrm{H}_{2}$ evolution reaction rates were quantitatively reduced by the mass $\%$ of polymer coating. In the case of the 10 mass $\%$ coated sample, there was no corrosion of $\mathrm{Al}$ nanoparticles. This fact suggested that the electrochemical reaction was suppressed by the polymer coating. Moreover, the reaction rate of $\mathrm{Al}$ nanoparticles was suppressed in proportion to the coverage percentage of the coating. Thus, to conclude, it was found that the corrosion rate of $\mathrm{Al}$ nanoparticles could be quantitatively suppressed by the coverage percentage of the polymer coating.
\end{abstract}

Keywords: corrosion; nanoparticle; aluminum; reaction; coating; polymer 


\section{Introduction}

In general, nanoscience is a well developed area with respect to synthesis, characterization, exploration, and exploitation of nanostructured materials. These materials are characterized as having at least one side of length in the nanometer range. Thus, the surface area of nanomaterials is increased remarkably compared with bulk materials, enabling them to have dramatically increased surface reaction rates. From the point of view of high surface area materials, the size effect of nanomaterials has been investigated in the fields of catalysis [1-5], sensors [6-8] and batteries [9,10].

Although the reaction rate can be increased by making the particle nano-sized, however then, the deterioration of the nano metal can be caused by corrosion under the atmosphere. In general, the nanoparticles need to be stored as raw materials for many production processes. Then, corrosion problems usually occur under high humidity conditions. It is a problem that corrosion reduces the quality of nanoparticles by forming oxides.

Moreover, in the case of coastal areas, the air borne salt particles coming from the sea, cause severe corrosion conditions. Besides, the film thickness of the coating should be extremely thin because each nanoparticle needs to be coated separately without bonding to one another in the synthesis. Although it is necessary to prevent the corrosion of metal nanoparticles, there are few papers on this problem.

In this paper, we have attempted to prevent the corrosion of aluminum (Al) nanoparticles using a polyurethane polymer coating. The coverage percentage of the polymer coating was varied from 0 to $100 \%$ to investigate the corrosion behavior of the nanometals quantitatively. The coverage film was investigated by nano-level investigation using TEM and AFM. Then, the corrosion reaction was examined for the polymer coated nano $\mathrm{Al}$ on a Pt plate by a wet and dry test using a $\mathrm{NaCl}$ solution [11]. Using these results, the relationship between the coverage percentage of polymer and the corrosion rate of the nanometal was discussed. Finally the corrosion prevention mechanism of the nanometal was investigated by changing the coverage percentage of the polymer film quantitatively.

\section{Experimental Procedure}

\subsection{Creation of Polymer Coated Al Nanoparticles}

The Al nanoparticles were prepared using an atomizing method. Melted Al was atomized and sieved as particles 300-600 nanometers (nm) in diameter. The Al surface oxide was completely removed in $0.5 \mathrm{M} \mathrm{H}_{2} \mathrm{SO}_{4}$ solution, thus markedly improving the surface activity. Moreover, this process is very important for conducting the synthesis of the polymer only on the metal nanoparticles.

The polyurethane polymer was synthesized on the Al nanoparticle by the chemical reaction of isocyanate and polyol as shown in Figures 1 and 2. Fluorine $(\mathrm{F})$ was found as either $\mathrm{CF}_{2}$ or $\mathrm{CHF}$ in the polyol. The urethane polymer was dissolved in isopropyl alcohol with the Al nanoparticles, and sufficiently agitated to wet the surface of the nanoparticles. After the isopropyl alcohol was completely evaporated under vacuum, the polyurethane polymer was hardened at atmospheric pressure. Then, the polymer was completely consumed to make a thin film on the nanoparticles. The mass $\%$ ratios of the polymer to $\mathrm{Al}$ in the synthesis were $0 \%, 0.1 \%, 0.4 \%, 1.0 \%, 3.0 \%$ and $10.0 \%$, in order to change the coverage percentage of polymer film on the surface of the nanoparticle. As is explained below, the coverage percentage was only able to be controlled by the mass \% ratio of polymer in the synthesis. 
Figure 1. Schematic diagram of the synthesis reaction for the polymer on Al nanoparticles.

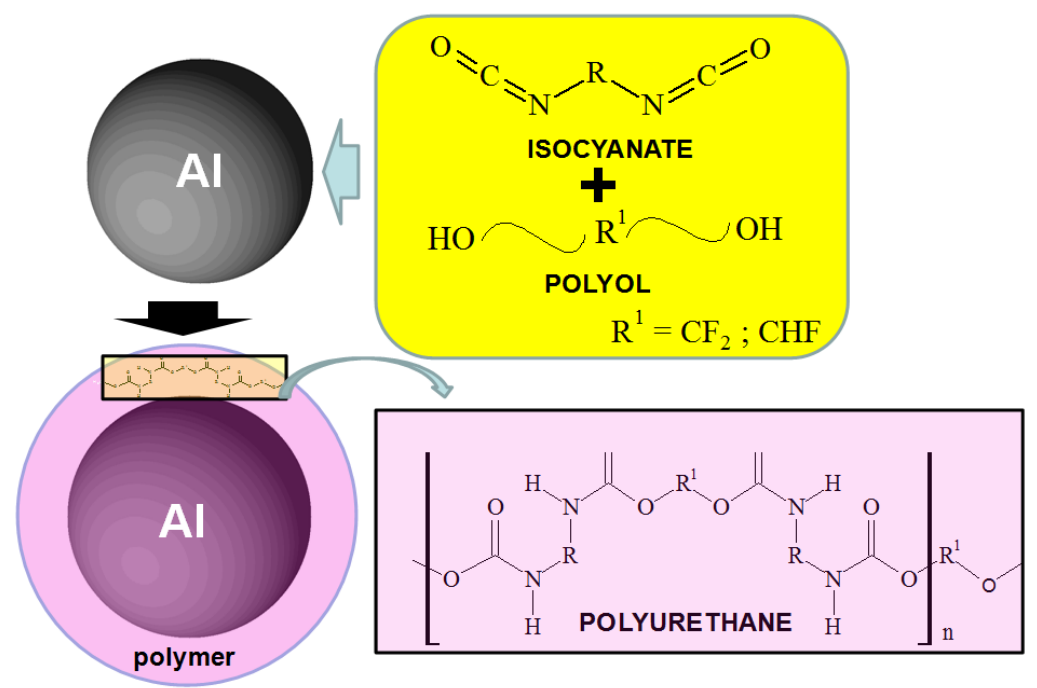

Figure 2. Schematic diagram of the wet and dry corrosion test.
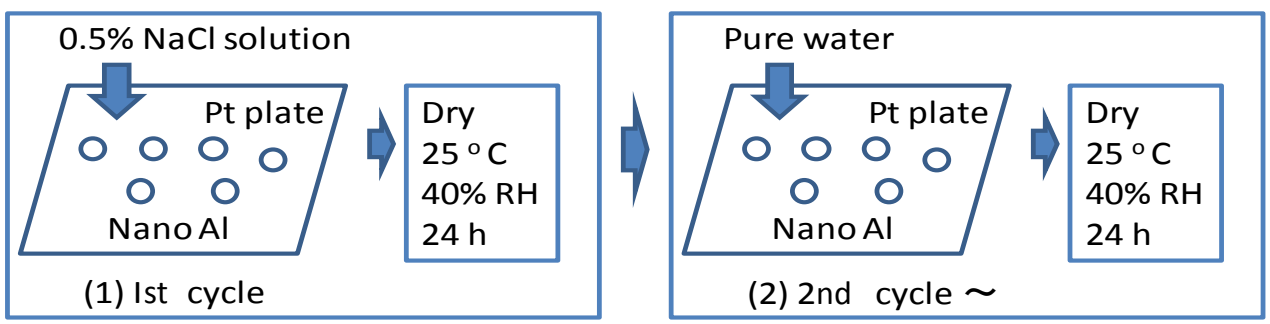

\subsection{Surface Analysis of the Polymer Film on Nano Al}

As for TEM, Energy Dispersive X-ray Spectroscopy (EDXS) was used to estimate the coverage percentage of the polymer on the $\mathrm{Al}$ nanoparticles by measuring the amount of $\mathrm{F}$ in the polymer as a marker. In order to investigate the chemical state of the polymer film in more detail, Electron Energy Loss Spectroscopy (EELS) spectra were observed by TEM [12]. The accelerated voltage was $200 \mathrm{kV}$, and the half width of value of zero loss was $1 \mathrm{eV}$. Moreover the nano level investigation on the sample was conducted by Atomic Force Microscopy (AFM) and Kelvin Force Microscopy (KFM) measurements. The machine for AFM/KFM is a SPA400 (Seiko Instruments SII with SPI 3800N probe station, Chiba, Japan). First, the Al nanoparticles were dispersed in methanol, ultrasonicated for $5 \mathrm{~min}$, placed on a polished Pt plate, and then AFM and KFM investigations were begun. In the AFM measurement, the surface morphology and potential distribution (KFM) measurements were conducted simultaneously with the tapping mode using gold coated cantilevers with a spring constant of $2.1 \mathrm{~N} / \mathrm{m}$.

\subsection{Corrosion Analysis}

Wet and dry corrosion tests (Figure 2) were conducted for 20 days in a 0.5 mass $\% \mathrm{NaCl}$ solution for 20 days (cycles) to estimate the corrosion behavior of the polymer coated $\mathrm{Al}$ nanoparticles. Only for the first cycle of testing, a 0.5 mass $\% \mathrm{NaCl}$ solution was poured over the $\mathrm{Al}$ nanoparticles on the platinum (Pt) plate in a chamber kept at $40 \%$ relative humidity $(\mathrm{RH})$ and $25^{\circ} \mathrm{C}$. Under this condition, the sample 
was dried for several hours. Then, from the 2 nd cycle of the test, pure water was poured over the samples under the same conditions every $24 \mathrm{~h}$. As the $\mathrm{NaCl}$ remains on the Pt plate, it has corrosive conditions after the 2 nd cycle of the test. During the corrosion test the samples are not removed.

There is no corrosion of $\mathrm{Al}$ nanoparticle without contact with the Pt plate. However, the anodic dissolution of $\mathrm{Al}$ occurs by galvanic contact with $\mathrm{Pt}$ which has a large cathodic area. Finally, in order to evaluate the corrosion rate, the sizes of the $\mathrm{Al}$ nanoparticles were measured after a wet and dry corrosion test. The size of the $\mathrm{Al}$ nanoparticles was normalized for 20 samples by the supposition that the average of the initial diameter of particles was $100 \%$.

After the corrosion test, the corrosion behavior of the nanoparticles was observed by SEM, AFM and KFM. It was found that the corrosion rate was suppressed in proportion to the coverage percentage of the polymer coating. Finally, a corrosion prevention mechanism for the nanometal was demonstrated by changing the surface coverage percentage of polymer coating quantitatively.

Besides this, the effect of polymer coating mass \% on the corrosion of $\mathrm{Al}$ nanoparticles in an alkali solution was examined. The volume of $\mathrm{H}_{2}$ evolution for $70 \mathrm{mg}$ of $\mathrm{Al}$ nanoparticles was measured in a $0.5 \mathrm{M} \mathrm{NaOH}$ solution. $\mathrm{H}_{2}$ gas was collected using a glass apparatus in the solution. In order to reduce problems in the experiment, the test time was shortened.

\section{Results and Discussion}

\subsection{Surface Analysis of the Polymer Coated Al Nanoparticles}

Figure 3 shows the secondary electron image of the 3 mass\% polymer coated $\mathrm{Al}$ nanoparticles by SEM. Here, 3 mass \% means that the polymer is coated as a weight ratio of 3 mass\% against the weight of Al. It was found that the particle diameter was 300-600 nm, and the particles were partially covered by the polymer. In order to investigate the actual mass\% of polymer to Al nanoparticles, thermo-gravimetric analysis (TGA) was conducted under a $\mathrm{N}_{2}$ atmosphere (Figure 4). As the temperature was increased above $200{ }^{\circ} \mathrm{C}$, the weight of the nanoparticle decreased due to the melt of the polymer. The weight loss of the 1, 3 and 10 mass\% coated $\mathrm{Al}$ nanoparticles was 1.3, 3.6 and 8.8 mass\%, respectively, as measured with TGA. As the sensitivity of TGA is not high, the weight loss from the Al nanoparticles is almost the same as the mass\% initially used in the synthesis. Thus, almost all of the polymer synthesized remains on the surface of the nanoparticles. It is possible to control the mass $\%$ of actual polymer coating using the mass \% of polymer to $\mathrm{Al}$ in the synthesis.

In order to investigate the coating state of the polyurethane polymer in more detail, TEM images were made as shown in Figure 5. For the 10 mass\% coated sample, a $10 \mathrm{~nm}$ thick polymer uniformly coats the entire surface of the $\mathrm{Al}$ nanoparticles. Thus the amount of 10 mass\% of polymer coating is thought to be enough to cover all of the surface of the Al nanoparticle. On the other hand, the 3 mass\% sample is only partially coated. However, the thickness of the polymer is also $10 \mathrm{~nm}$ in the areas where the particles are coated. In fact, the polymer thickness was almost $10 \mathrm{~nm}$ in all of the polymer samples. Although a thickness of $10 \mathrm{~nm}$ is perhaps needed in order to maintain the film, the detailed mechanism is unknown. In this way, as the film thickness is very thin, each nanoparticle is able to be coated separately without bonding to one another in the synthesis. This is very important to coat the nanometal while maintaining its characteristics. 
Figure 3. The secondary electron image of the 3 mass $\%$ polymer coated Al nanoparticles by Scanning Electron Microscopy (SEM).

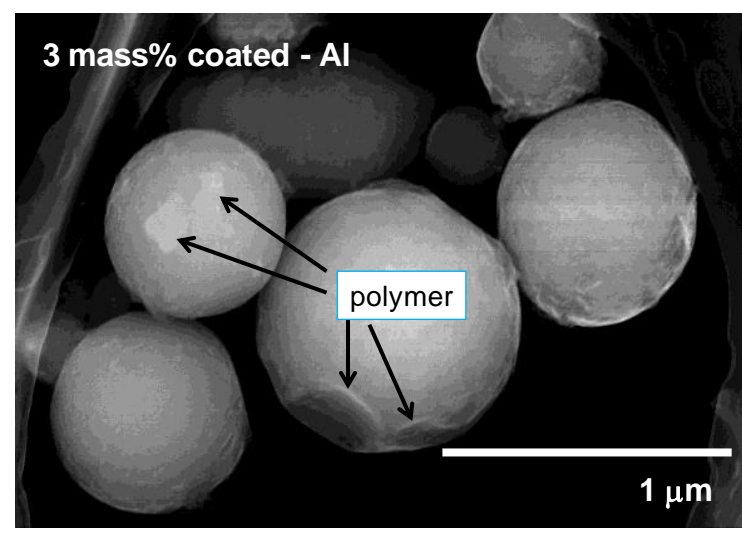

Figure 4. Thermo Gravimetric Analysis (TGA) of the coated Al nanoparticles.

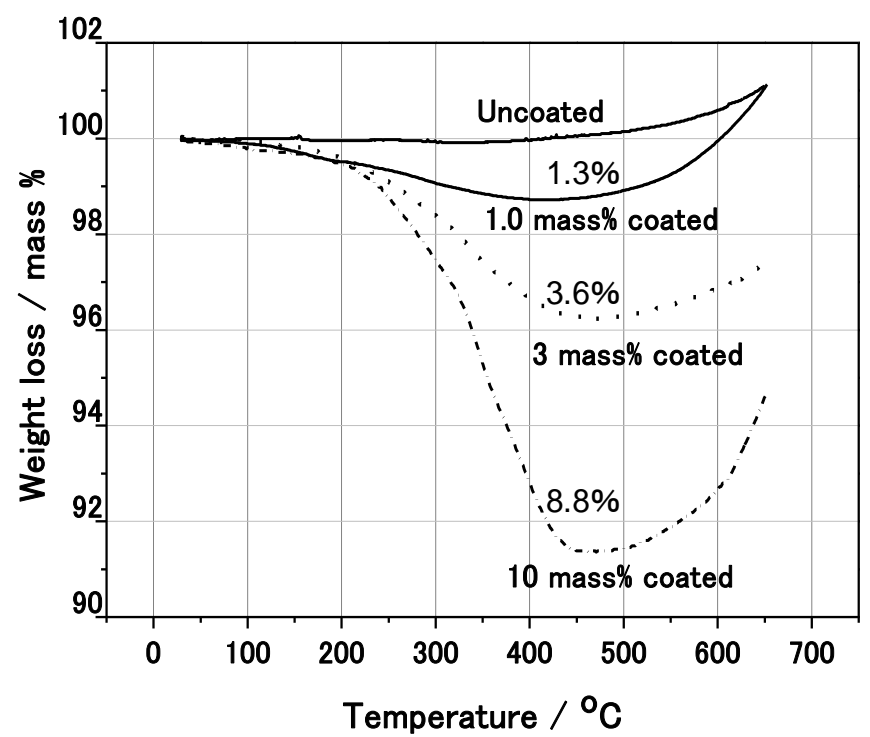

Figure 5. Transmission Electron Microscopy (TEM) images of the 3 mass $\%$ and 10 mass $\%$ coated Al nanoparticles.
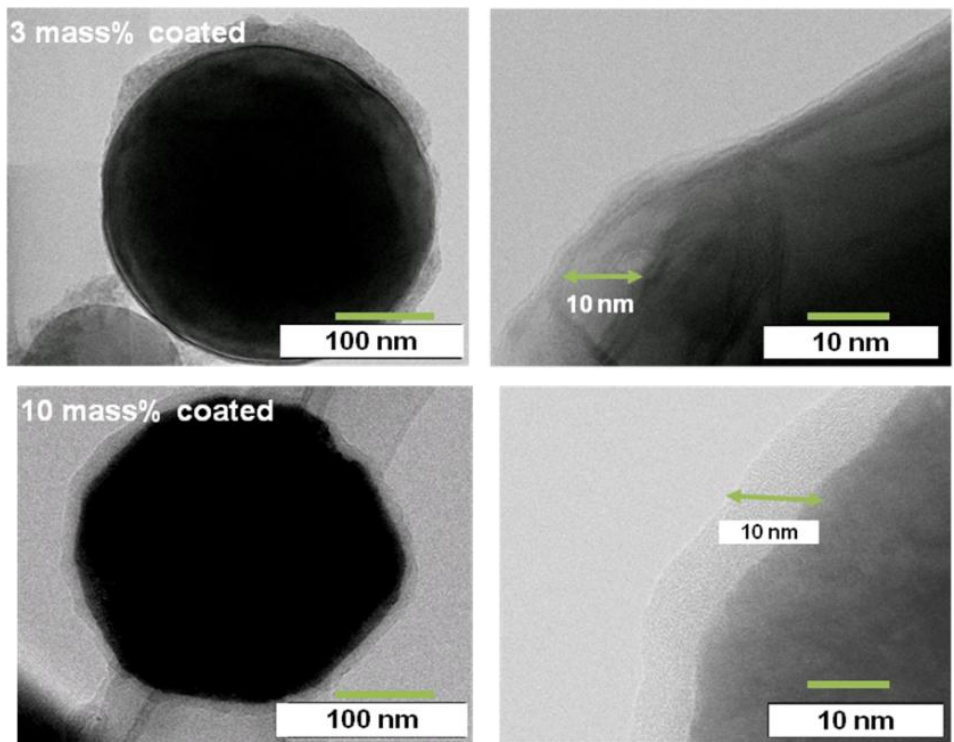
The EDXS mappings were measured to estimate the coverage state of the film coating on the $\mathrm{Al}$ nanoparticle. The EDXS concentration of fluorine (F) was higher in the case of the 10 mass\% coated sample than the 3 mass $\%$ coated one. In Figure 6 the EDXS count of F increases linearly as the mass $\%$ of the coating increases. Since $\mathrm{F}$ is contained in the polymer as $\mathrm{CF}_{2}$ or $\mathrm{CHF}$, the EDXS count of $\mathrm{F}$ is proportional to the coating coverage percentage. Thus, the coating coverage percentage can be practically estimated from the EDXS F counts. As for the 10 mass $\%$ sample, $100 \%$ coverage is identified by the TEM in Figure 6, and the count is almost 200. Thus, the coverage percentage for the 3 mass $\%$ sample is estimated to be almost $40 \%$ from an F count of 90 . Similarly, the coverage percentage for the 1 mass\% sample is estimated to be almost $25 \%$ from an F count of 50 . In this way, the coverage percentage of film coating is able to be practically estimated by the EDXS counts of F. Thus, it has been demonstrated that the coverage percentage of the developed samples can be controlled from 0 to $100 \%$ by changing the mass $\%$ of polymer coating in the synthesis. In addition, it has been found that the relationship between the film coverage percentage and the coating mass $\%$ can be obtained by TEM analysis.

Figure 6. Energy Dispersive X-ray Spectroscopy (EDXS) analysis of $\mathrm{F}$ and $\mathrm{Al}$ for the $\mathrm{Al}$ nanoparticles, and coverage percentage of the film coating calculated by EDXS counts.
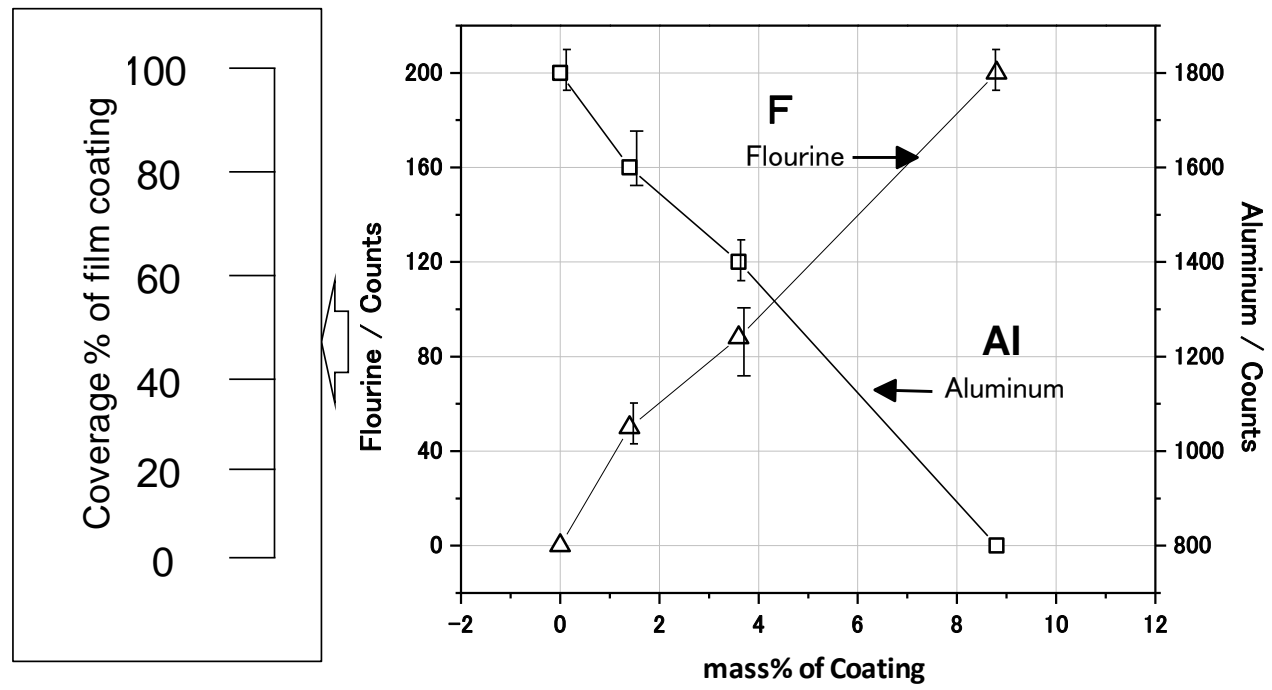

The Electron Energy Loss spectroscopy (EELS) spectra were obtained for the 10 mass $\%$ coated Al to estimate the chemical state of $\mathrm{C}, \mathrm{N}$, and $\mathrm{O}$ in the polymer film in Figure 7. There are strong peaks for $\mathrm{C}-\mathrm{K}$ at $284, \mathrm{~N}-\mathrm{K}$ at 410 and $\mathrm{O}-\mathrm{K}$ at $535 \mathrm{eV}$. On the other hand, there is a very weak peak for F-K at $690 \mathrm{eV}$. The spectrum of C-K shows the typical peak showing the $\sigma$-bond. These peaks of C-K, N-K and $\mathrm{O}-\mathrm{K}$ are thought to show the chemical bond of the polyurethane polymer. The Bright field (BF) image (a) and EELS mappings are shown in (b) C-K, (c) O-K and (d) F-K for the 10 mass\% coated Al in Figure 8 . The $\mathrm{BF}$ image shows a polymer film of almost $10 \mathrm{~nm}$. The high concentration of $\mathrm{C}$ and $\mathrm{O}$ are shown in the polymer film. However, the concentration of $\mathrm{C}$ and $\mathrm{O}$ are very low on the particle because the thickness of the $\mathrm{Al}$ particle is very high. Thus, the high concentration of $\mathrm{C}-\mathrm{K}$ and $\mathrm{O}-\mathrm{K}$ in the film is thought to show the chemical bond of polyurethane polymer. The mapping of $\mathrm{F}$ is not detected clearly because the peak strength is weak. The EELS spectra are detected only from the film outside of the particle, which is a very narrow area. On the other hand, EDXS are taken from all over the film on the particle. Thus the sensitivity and the reliability in EDXS for F are much higher than in EELS. 
Figure 7. Electron Energy Loss spectroscopy (EELS) spectra of (a) C-K and (b) N-K, O-K for 10 mass $\%$ coated Al.
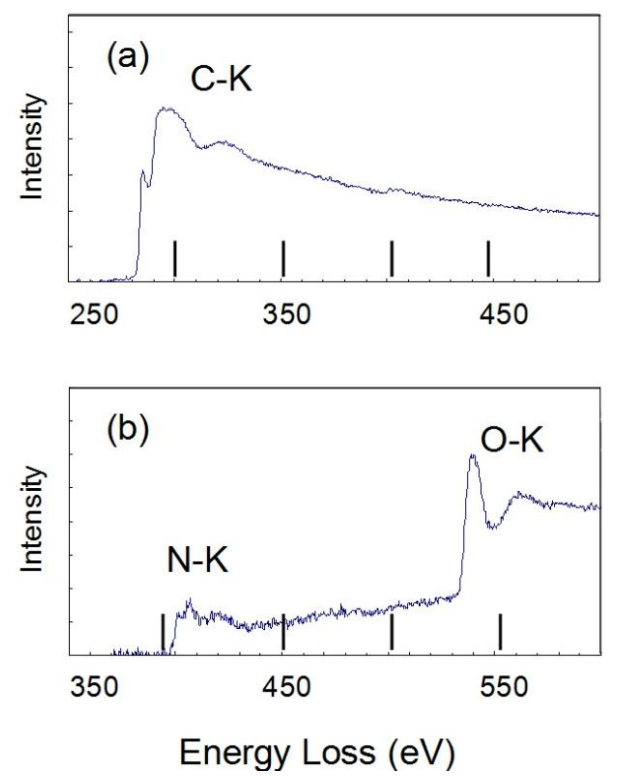

Figure 8. (a) Bright field image; and EELS mappings of (b) C-K, (c) O-K, (d) F-K for 10 mass $\%$ coated Al.
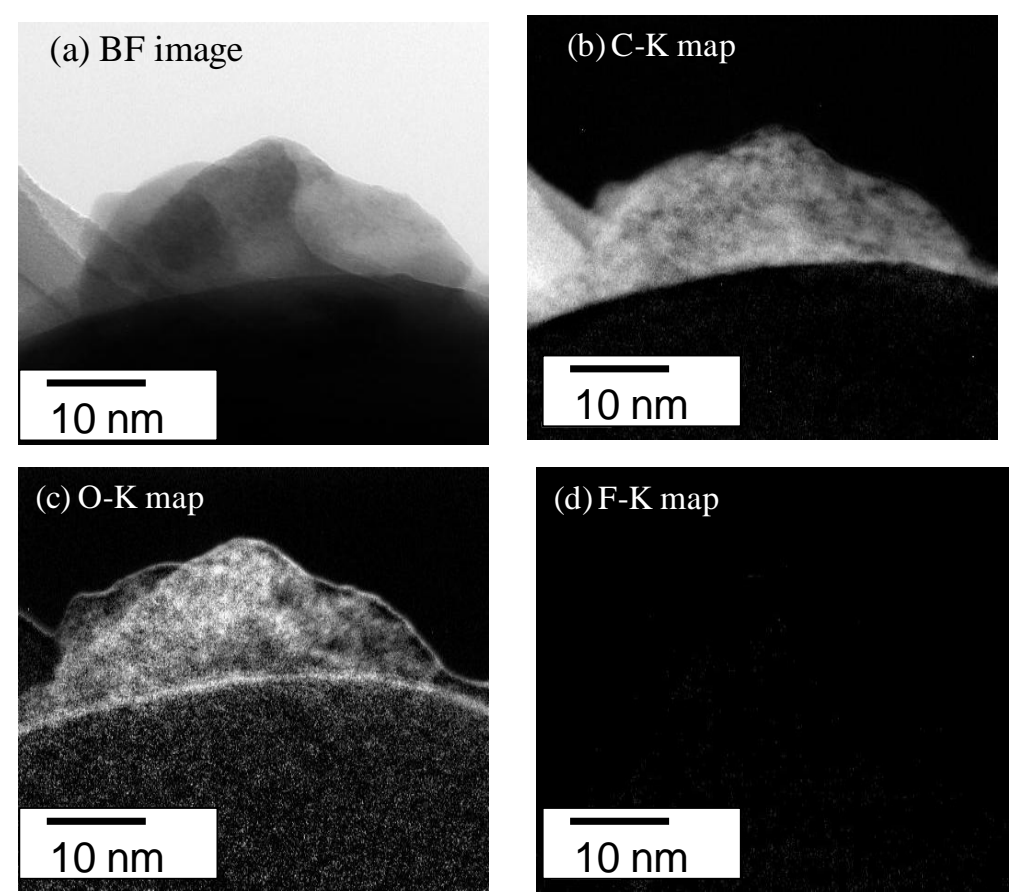

concentration

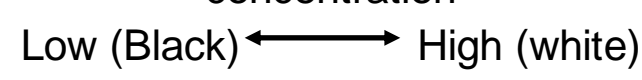

\subsection{Corrosion of the Polymer-Coated Al Nanoparticles}

AFM and KFM (potential distribution) measurements were carried out using the tapping mode as shown in Figure 9. The KFM image for the uncoated Al particle is represented by a half globe, almost the same as the AFM image. On the other hand, although the AFM images for the 3 mass $\%$ coated Al 
particle is a half globe, the KFM image displays a broken shape. Thus, the potential around the Al particles is thought to be partially isolated from the Pt plate by the polymer coating. This fact suggests that the electrochemical reaction is suppressed by the coverage of the polymer. In other words, it is possible to prevent the corrosion reaction by coverage of the polymer film.

Figure 9. Atomic Force Microscopy (AFM) and Kelvin Force Microscopy (KFM) (the potential distribution) images of $\mathrm{Al}$ nanoparticles as 3-dimensional representations. (a) AFM images of the nanoparticle of uncoated $\mathrm{Al}$ (1) and a 3 mass\% polymer coated $\mathrm{Al}$ (2); (b) KFM images of uncoated $\mathrm{Al}$ (1) and a 3 mass \% polymer coated $\mathrm{Al}$ (2).
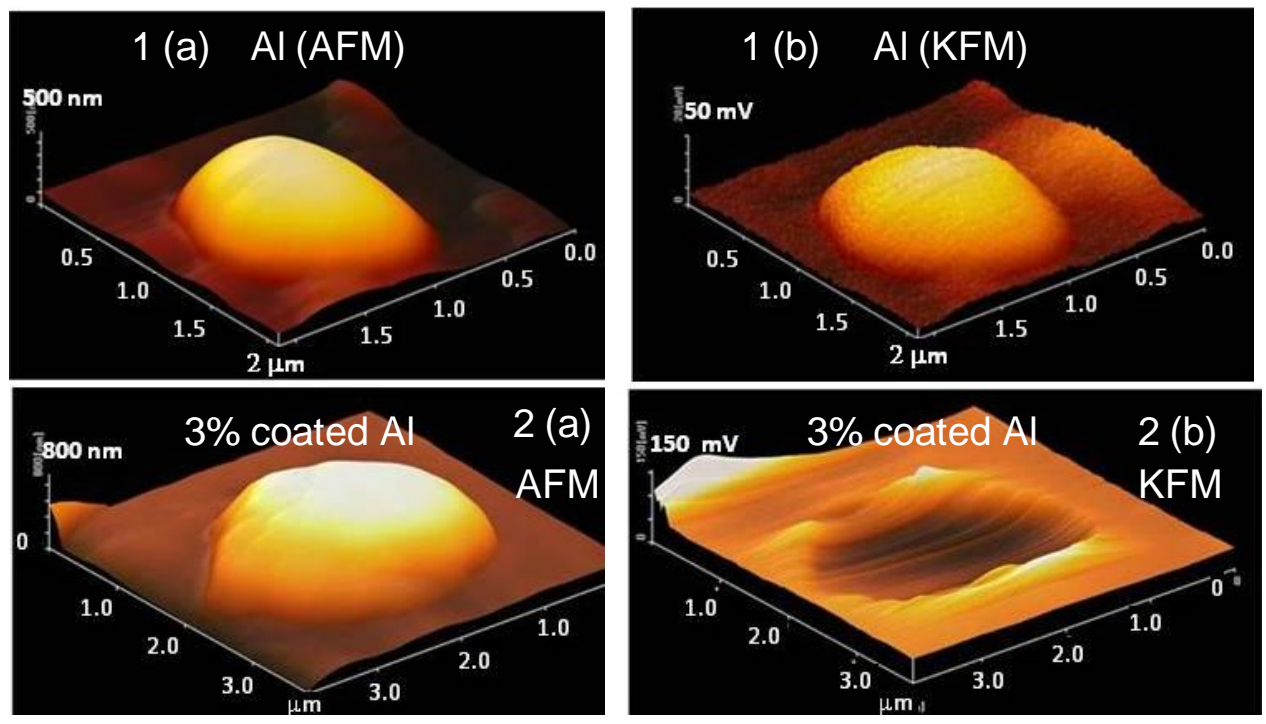

A wet and dry corrosion test using a $\mathrm{NaCl}$ solution was conducted. A 0.5 mass\% $\mathrm{NaCl}$ solution was poured over the $\mathrm{Al}$ nanoparticles in a chamber kept at $40 \%$ relative humidity $(\mathrm{RH})$ and $25^{\circ} \mathrm{C}$. Figure 10 shows SEM images of the Al nanoparticles taken after the corrosion test at each day. The shape of the 10 mass \% polymer coated sample is unchanged after 14 days, showing that it has not been subjected to corrosion. This corrosion prevention is due to complete coverage with polymer which isolates $\mathrm{Al}$ particles from the Pt plate. However, the 3 mass $\%$ sample is partially corroded at 7 days, and continues to be corroded, yielding a slender-shaped particle after 14 days. Actually, the diameter of the particle is reduced by $20 \%$ at the area of the highest corrosion at 7 days. Additionally, it is reduced by $60 \%$ at 14 days. In this case, the corrosion is thought to start in an area of bare (no polymer) metal, and the electrochemical reaction continues in that region. Finally the galvanic corrosion between $\mathrm{Al}$ particle and the Pt plate is thought to continue for 14 days. In this way, the shape of the Al nanoparticle varies with the polymer coating percentage after the corrosion test.

AFM and KFM measurements were carried out for uncoated Al nanoparticles after the corrosion test in Figure 11. The KFM image for the uncoated Al particle at day zero is represented by a half globe, almost the same as the AFM image. On the other hand, although the AFM images for uncoated Al particle at day one are half globes, the KFM images display broken shapes. For example, the top (highest) potential in KFM is changed from $18,7,1 \mathrm{mV}$ at day $0,1,20$, respectively. Thus, the potential value around the $\mathrm{Al}$ particles is thought to be very low showing the start of the electrochemical reaction by galvanic corrosion between the Al particle and the Pt plate. In the same way, the KFM image for the 
uncoated sample at 20 days display a broken shape. This fact suggests that the electrochemical reaction of a sample continues to 20 days. In this way, it is possible to measure the corrosion reaction of nanoparticles precisely by AFM and KFM.

Figure 10. SEM images of Al nanoparticles subjected to a wet and dry corrosion test in Figure 2.

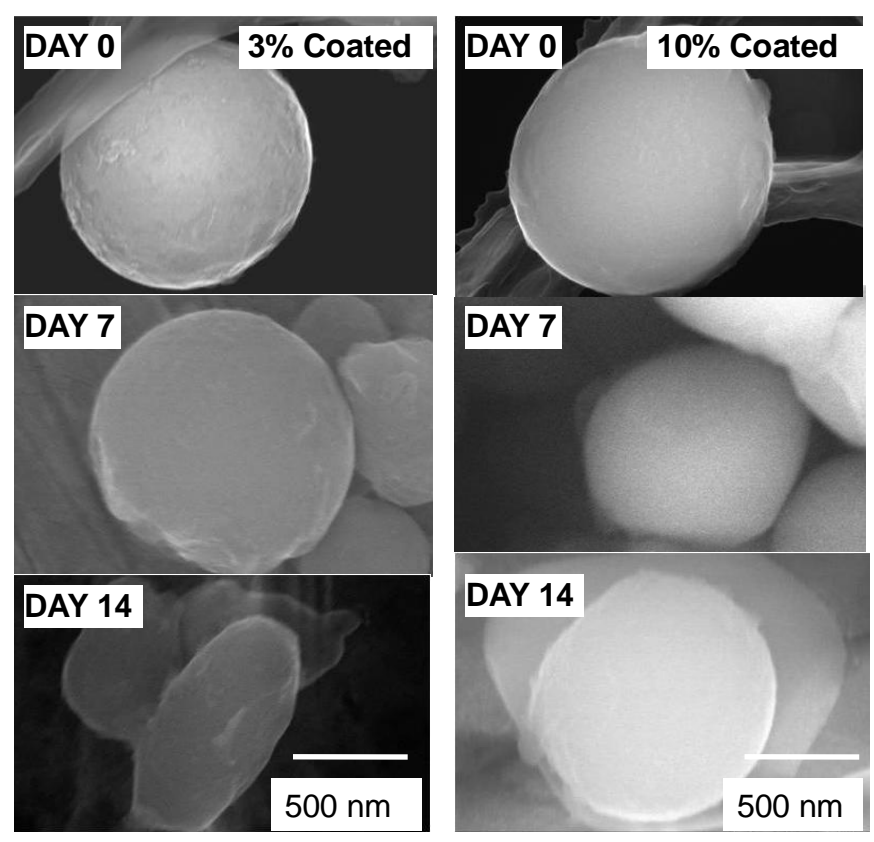

Figure 11. (a) AFM and (b) KFM images of uncoated $\mathrm{Al}$ nanoparticles subjected to corrosion test for day 0 , day 1 , day 20 .
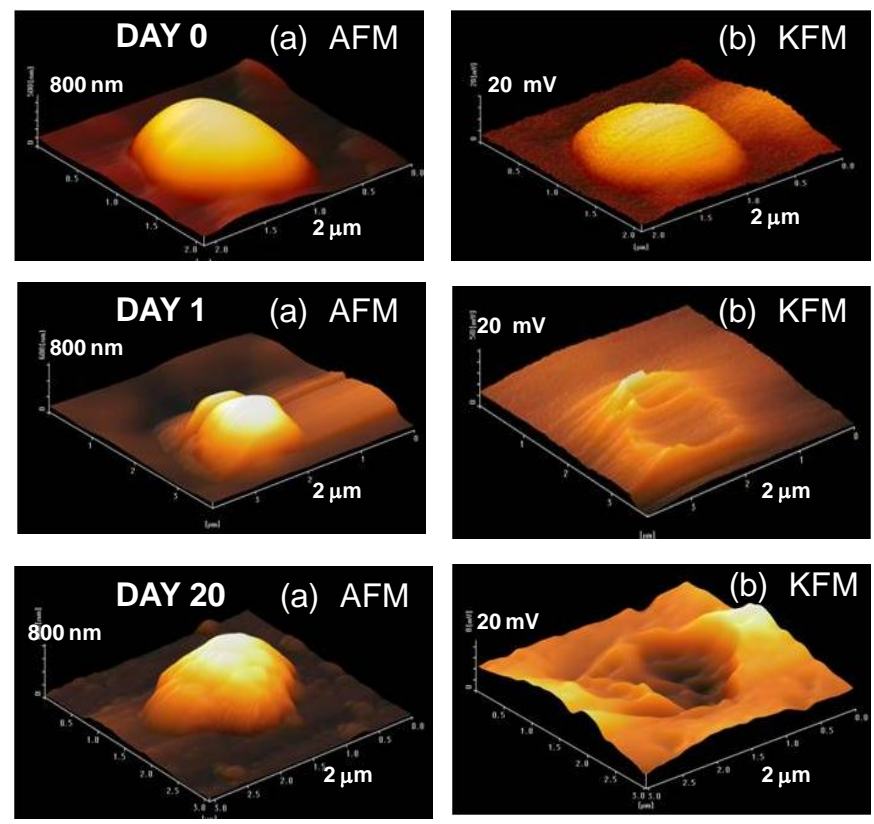

Figure 12 shows the effect of the coating mass \% on the sizes of the Al nanoparticles after a wet and dry $\mathrm{NaCl}$ corrosion test. The size of the $\mathrm{Al}$ nanoparticles was normalized for the 20 samples by the supposition that the average of the initial diameter of the particles was $100 \%$. The normalized size $\left(\mathrm{S}_{\mathrm{N}}\right)$ 
of uncoated $\mathrm{Al}$ decreases remarkably with time in the corrosion test, showing that the corrosion rate is very high. On the other hand, the $\mathrm{S}_{\mathrm{N}}$ of the 10 mass\% coated $\mathrm{Al}$ hardly changes. This fact suggests that corrosion is completely prevented by the polymer film in the case of the 10 mass $\%$ coated Al. After 14 days, the $S_{N}$ of the $0,1.0,3.0$, and 10.0 mass $\%$ coated samples are $63 \%, 74 \%, 87 \%$ and $97 \%$, respectively. Thus, the $\mathrm{S}_{\mathrm{N}}$ after 14 days shows a higher value as the coating mass $\%$ increases. It was found that the corrosion reaction is suppressed in proportion to the mass \% of polymer coating.

Figure 13 shows the effect of polymer coating mass $\%$ on the corrosion of $\mathrm{Al}$ nanoparticles in an alkali solution. The volume of $\mathrm{H}_{2}$ evolution reaction for $70 \mathrm{mg}$ of $\mathrm{Al}$ nanoparticles was measured in a $0.5 \mathrm{M} \mathrm{NaOH}$ solution. The volume of $\mathrm{H}_{2}$ evolving for each sample increases with the test time in the figure. After $120 \mathrm{~s}$, the 0.1, 0.4, 3.0 and 10 mass\% coated $\mathrm{Al}$ has an evolution volume of 24, 21, 6, and $1 \mathrm{~mL}$, respectively. Thus, less $\mathrm{H}_{2}$ evolution is shown as the coating mass\% increases, which is just the same as the previous wet and dry corrosion test in Figure 12. In this way, the $\mathrm{H}_{2}$ evolution reaction was also suppressed in proportion to the mass $\%$ of polymer coating.

Figure 12. Effect of coating mass $\%$ on the sizes of $\mathrm{Al}$ nanoparticles caused by the wet and dry corrosion test with $\mathrm{NaCl}$.

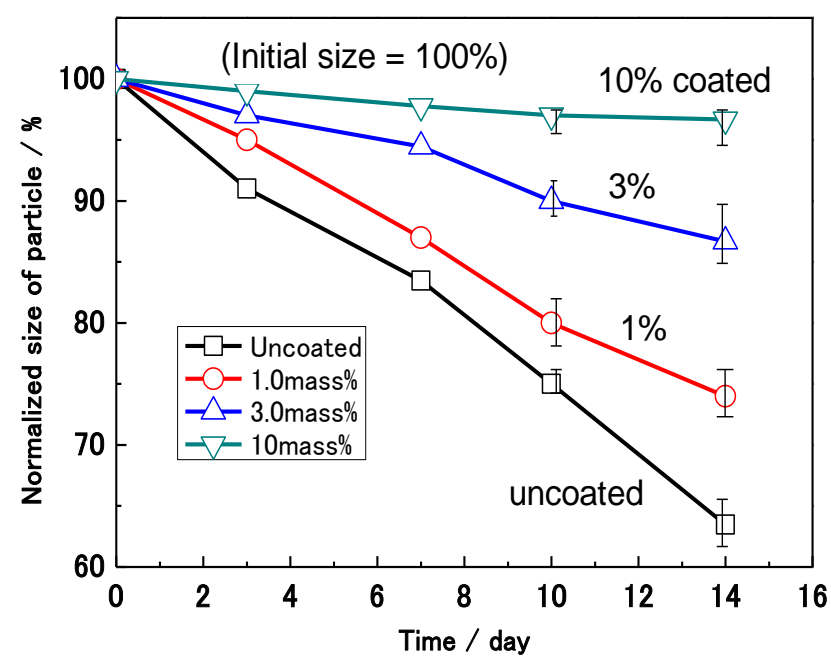

Figure 13. Effect of coating mass $\%$ on the hydrogen $\left(\mathrm{H}_{2}\right)$ evolution reaction on $\mathrm{Al}$ nanoparticles.

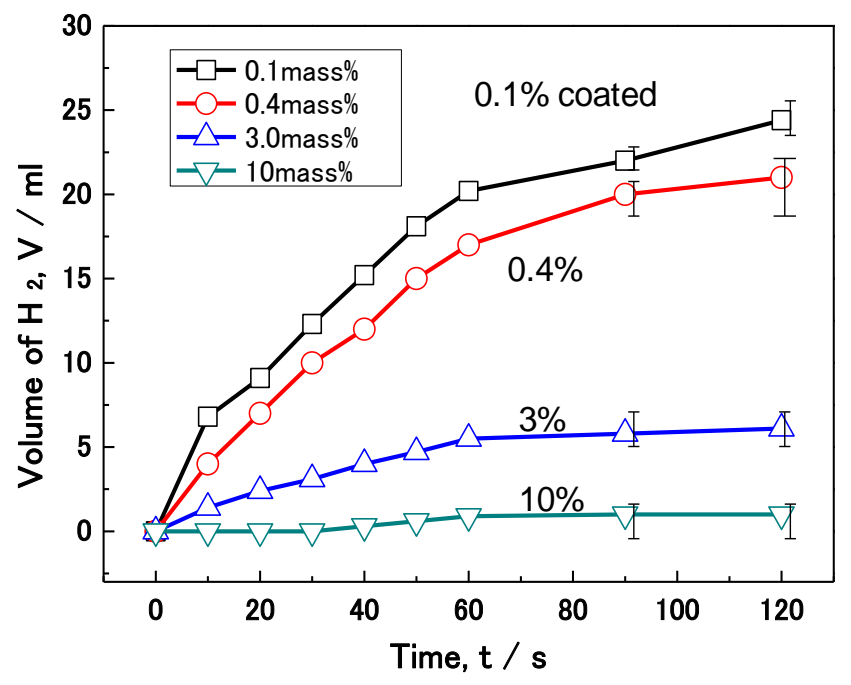


Figure 14 summarizes the effect of mass $\%$ of polymer coating on the reaction rate in each experiment from Figures 12 and 13. The rate of corrosion for each coated sample was determined by the supposition that the amount of corrosion of uncoated $\mathrm{Al}$ at 14 days was $100 \%$. In the same way, the rate of the $\mathrm{H}_{2}$ reaction was determined by the supposition that the volume of uncoated $\mathrm{Al}$ at $120 \mathrm{~s}$ was $100 \%$. Both rates were reduced as the mass $\%$ of polymer coating increased. This fact indicates that corrosion reactions on the $\mathrm{Al}$ particles under these conditions are suppressed by the polymer coating. Moreover, a quantitative relationship between the corrosion rate and the mass $\%$ of coating was established. The film coating coverage percentage was determined by the results of EDAX from TEM (Figure 6). It was also demonstrated that the corrosion rate of $\mathrm{Al}$ nanoparticles was suppressed in proportion to the coverage percentage of the polymer coating.

Figure 14. Effect of mass $\%$ of coating on the reaction rate of $\mathrm{H}_{2}$ evolution and corrosion from Figures 12 and 13. Mass\% of coating was changed to coverage percentage of film coating by TEM analysis.

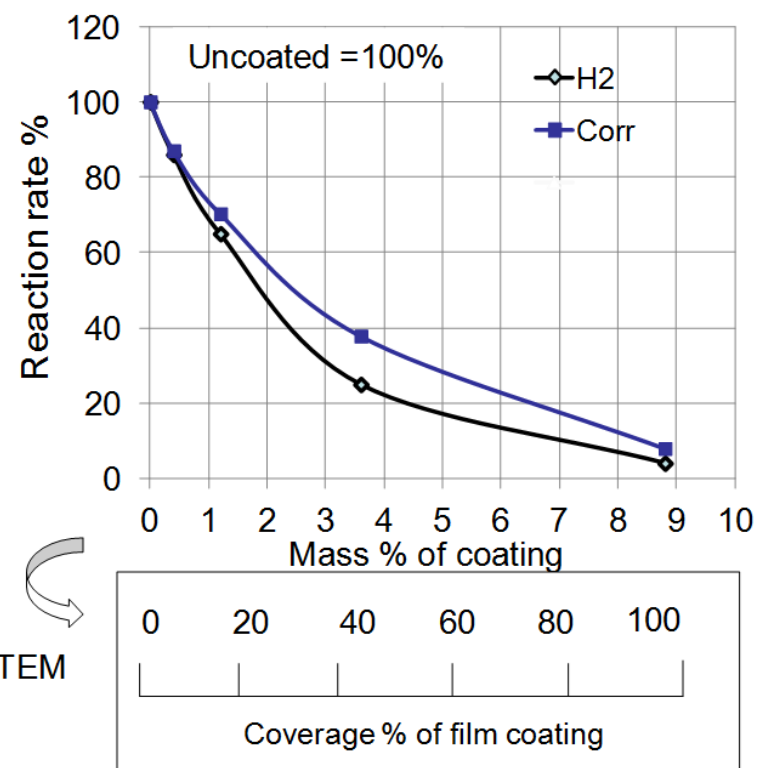

From these experiments, the corrosion rate of $\mathrm{Al}$ nanoparticles was able to be quantitatively controlled by the surface coverage percentage of the polymer coating. Moreover, almost all of the polymer synthesized was consumed in the formation of the surface film. In other words, we can control the polymer coating mass $\%$ using the mass $\%$ of polymer in the synthesis. In addition, the film thickness was almost the same $-10 \mathrm{~nm}$ for each coating. Thus, we can control the coverage percentage of polymer coating very easily from the mass\% of polymer in the synthesis. Besides, as the film thickness is very thin, each nanoparticle is able to be coated separately without bonding to one another in the synthesis. In this way, the coating of a nanometal can be established using only the mass \% ratio of polymer to metal in the synthesis.

Finally, it can be said that we can prevent the corrosion of nanometals by using a polyurethane polymer coating. Thus, it is possible to store nanometals without corrosion in the atmosphere at high humidity. 


\section{Conclusions}

In order to prevent corrosion, aluminum nanoparticles were coated with polyurethane polymer.

Using the EDAX analysis for $\mathrm{F}$ in the polymer by TEM, the coverage percentage of each coating was able to be determined. The coverage percentage of the 10 mass $\%$ and 3 mass $\%$ polymer coated $\mathrm{Al}$ particles in the synthesis was almost $100 \%$ and $40 \%$, respectively. However, the film thickness was almost $10 \mathrm{~nm}$ for both coatings. As the film thickness was very thin, each nanoparticle was able to be coated separately without bonding to one another in the synthesis.

In AFM, the potential around the $\mathrm{Al}$ particles showed a lower value with increased polymer coating, indicating that the conductivity of the nano $\mathrm{Al}$ was isolated from the Pt plate by the polymer. The reaction rates for both wet/dry and $\mathrm{H}_{2}$ evolution tests were reduced as the mass $\%$ of polymer coating increased. Thus, the corrosion reaction on the Al particles was quantitatively suppressed by the polymer coating.

The corrosion rate of a nanometal could be reduced completely by using a polymer coating on the surface. Thus, it is possible to keep nanometals without corrosion in the atmosphere at high humidity.

\section{Author Contributions}

Toshiyasu Nishimura made the polymer coated Al nanoparticles, and conducted surface analysis. Vedarajan Raman conducted the corrosion test and the observation of nanoparticles by using AFM and KFM.

\section{Conflicts of Interest}

The authors declare no conflict of interest

\section{References}

1. Bond, G.C. The origins of particle size effects in heterogeneous catalysis. Surf. Sci. 1985, 156, 966-981.

2. Sachtler, W.M.H. Morphology and crystal face specificity of catalysis on small particles. Ultramicroscopy 1986, 20, 135-143.

3. Haruta, M. Size- and support-dependency in the catalysis of gold. Catal. Today 1997, 3, 153-166.

4. Okumura, M. The reactivities of dimethylgold(III) $\beta$-diketone on the surface of $\mathrm{TiO}_{2}$ : A novel preparation method for Au catalysts. Solid State Ionics 1997, 95, 143-149.

5. Chan, K.Y.; Ding, J.; Ren, J.; Cheng, S.; Tsang, K.Y. Supported mixed metal nanoparticles as electrocatalysts in low temperature fuel cells. J. Matter. Chem. 2004, 14, 505-516.

6. Shimizu, Y.; Egashira, M. Basic aspects and challenges of semiconductor gas sensors. MRS Bull. 1999, 24, 18-24.

7. Tamaki, J. Grain-size effects in tungsten oxide-based sensor for nitrogen oxides. J. Electrochem. Soc. 1994, 141, 2207-2210.

8. Gurlo, A. Grain size control in nanocrystalline $\mathrm{In}_{2} \mathrm{O}_{3}$ semiconductor gas sensors. Sens. Actuators $B$ 1997, 44, 327-333. 
9. Fujimoto, H. Effect of crystallite size on the chemical compositions of the stage 1 alkali metal-graphite intercalation compounds. Carbon 1994, 32, 193-198.

10. Tokumitsu, K. Electrochemical insertion of lithium into carbon synthesized from condensed aromatics. J. Electrochem. Soc. 1996, 143, 2235-2239.

11. Nishimura, T. Corrosion behavior of silicon-bearing steel in a wet/dry environment containing chloride ions. Mater. Trans. 2007, 48, 1438-1443.

12. Nishimura, T. Electrochemical behavior and structure of rust formed on Si- and Al-bearing steel after atmospheric exposure. Corr. Sci. 2010, 52, 3609-3614.

(C) 2014 by the authors; licensee MDPI, Basel, Switzerland. This article is an open access article distributed under the terms and conditions of the Creative Commons Attribution license (http://creativecommons.org/licenses/by/3.0/). 